\title{
Chapter 3 \\ Future Implications of the Fourth \\ Industrial Revolution on Education \\ and Training
}

\author{
Paul Kim
}

The Fourth Industrial Revolution spurred many debates and discussions on how education sectors and industries can cope with the rapid pace of technological advancements and the implications for workforce and industry. In this regard, innovators have been pursuing development of learning technology solutions that could help society cope with these advancements. In particular, innovation leaders have been working on solutions to explore the potentials, and to leverage emerging technologies, of Artificial Intelligence (AI) and machine learning.

Countries such as the United States, France, the People's Republic of China (PRC), and those of the European Union have started including AI in their national strategies, investing in research, integrating it in their education systems, and preparing their workforce for an automated future. ${ }^{1}$ For instance, the Silicon Valley community in the United States is progressing fundamental research and development of AI in numerous directions, but the PRC has been making more significant headway in developing numerous AI-integrated applications with an unimaginably vast amount of data that they accumulate under much less stringent regulations. Considering these two differently competent camps, governments need to pursue prudent strategies to find a good balance between protecting privacy and fueling the advancement of AI to ultimately improve the lives of their own citizens.

\footnotetext{
${ }^{1}$ Kim, P. and Lee, J. (n.d.). Evolution of Online Learning Environments and the Emergence of Intelligent MOOCs.

\section{P. Kim (凶)}

Chief Technology Officer of Office of Innovation and Technology and Assistant Dean of Graduate School of Education, Stanford University, Stanford, CA, US

e-mail: phkim@stanford.edu
} 


\section{Issues and Challenges}

The rise of frontier technologies, including AI, is a disruptor that society should be prepared for. While AI holds immense potential in advancing societal developments, many organizations and companies are too slow to adapt and leverage the technology in their work. This can potentially cause huge challenges in addressing job-skills mismatch productivity and in preparing workers who may be affected by automation of jobs, filling new occupations created by emerging fields, and enabling small and medium-scale companies to adopt new technologies, among others.

\section{Proposed Solutions}

Many governments have been investing in lifelong learning as they anticipate and prepare for emerging skills and jobs. Aside from efforts in raising the quality of formal education systems, continuing education and retooling or upskilling have also been made more accessible through modalities such as work-based trainings, distance education, and massive open online courses, among others. To make these modalities efficient and effective, technologies are also being used and integrated in the classrooms as well as in various training programs.

Recently, there has been an increased volume of Education Technology (EdTech) solutions being released in the market because of the speed of advancement of technology. This is not surprising, as the value of investment in innovative EdTech companies is also increasing according to studies such as those by McKinsey \& Company in 2018 and Research and Markets in 2018. ${ }^{2}$ Most of these EdTech systems and solutions are integrating AI and deep learning models to maximize and enhance their existing services or create new possibilities toward better outcomes for students and teachers. $^{3}$

\section{Significant Examples of Good Practices}

\section{Teacher Advisor with Watson 1.0}

A free AI tool called Teacher Advisor with Watson 1.0 was developed by the IBM Foundation to help teachers strengthen their mathematics instruction. Teacher Advisor provides quality resources as well as instructional context and support to help teachers understand grade-level content in-depth, and enable them to use strategies that will be effective for their students. The AI tool, through its cognitive computing capabilities, can respond to teachers' queries and deliver the most relevant answers. IBM collaborated with the American Federation of Teachers, math teachers, and national education leaders to develop this resource. Its teaching materials are also

\footnotetext{
${ }^{2}$ Ibid.

${ }^{3}$ Ibid.
} 
vetted by a range of education experts and nonprofit entities including UnboundEd, Student Achievement Partners, Illustrative Mathematics, CPALMS, EngageNY, Achieve, and American Federation of Teachers Share My Lesson (Ascione 2017 as cited in Kim and Lee n.d.). ${ }^{4}$

Teacher Advisor helps ensure that teachers (i) save time finding quality resources by coming to a curated site for all of their planning needs, and (ii) have the context they need to implement those resources effectively for all students. ${ }^{5}$ It has recently been awarded as a Webby Honoree in two categories: Best Use of Machine Learning and Best Education Website.

\section{Georgia Tech's AI-Backed Teaching Assistant}

In a course at Georgia Tech, an AI-backed Teaching Assistant (TA), dubbed Jill Watson, made a debut by answering student questions and providing discussion topics (Goel and Polepeddi 2016). ${ }^{6}$ This teaching assistant was based on IBM's Watson platform. Jill was developed for an online Knowledge-Based Artificial Intelligence (KBAI) class, specifically to handle the high number of forum posts by students enrolled in an online course that is a requirement for Georgia Tech's online master of science in computer science program. ${ }^{7}$ The level of sophistication of this AI-based TA for student-question answering is still at an early stage, but with increasing data and the availability of deep learning models, the quality of system-generated responses is expected to improve over time. ${ }^{8}$

\section{PRC's AI-Enabled Education}

The PRC Government has made AI-enabled education a national strategy, consistent with its goal to make the country a global center of AI innovation by $2030 .{ }^{9}$ Given the amount of investments they have poured out and the volume of big data that they can organize, the PRC Government will most certainly become a key player in

\footnotetext{
${ }^{4}$ Ascione, L. (2017, October). New AI tool helps teachers tackle math. Retrieved from Eschoolnews Website: https://www.eschoolnews.com/2017/10/12/ibm-ai-tool-teachers-tackle-math/2/?all.

${ }^{5}$ IBM. (n.d.). Teacher Advisor With Watson. Retrieved from https://www.ibm.com/ibm/responsib ility/initiatives/activitykits/teacheradvisor/.

${ }^{6} \mathrm{Kim}$ and Lee (n.d.). op. cit.

${ }^{7}$ Lipko (2016) Meet Jill Watson: Georgia Tech's first AI teaching assistant. Retrieved from Georgia Tech Website: https://pe.gatech.edu/blog/meet-jill-watson-georgia-techs-first-ai-teachingassistant.

${ }^{8}$ Kim and Lee (n.d.). op. cit.

${ }^{9}$ Jing (2017. China wants to bring artificial intelligence to its classrooms to boost its education system. Retrieved from https://www.scmp.com/tech/science-research/article/2115271/chinawants-bring-artificial-intelligence-its-classrooms-boost.
} 
advancing $\mathrm{AI}$ in the near future. ${ }^{10}$ The PRC is even expected to overtake the United States in AI research.

Education has emerged as one of the hottest markets for the application of AI in the PRC based on market research. ${ }^{11}$ As a result, more online education companies have been looking into tapping AI to offer higher quality education and improve educational outcomes. For instance, a Shanghai-based educational platform called Master Learner uses AI to develop a "super teacher" to aid teachers in reviewing students' assignments and conduct diagnoses that will allow them to come up with personalized teaching plans. This super teacher is also capable of answering 500 million of the most tested questions in the PRC's middle schools. A company in the PRC also focused on boot camp education to train information technology workers to help address a shortage. These boot camps became so successful that they were able to have an initial public offering in the National Association of Securities Dealers Automated Quotations. ${ }^{12}$

\section{Allen Institute for Artificial Intelligence}

The Allen Institute for Artificial Intelligence (AI2) was created by Microsoft cofounder Paul Allen and renowned AI researcher Dr. Oren Erzioni to develop AI research and engineering for the common good. Aside from pursuing world-class research and development on $\mathrm{AI}$, it also undertakes projects that create open-source data and resources for the community, holds activities that foster breakthroughs, and supports underexplored but critical research.

One of AI2's notable projects is ARISTO, an intelligent system that reads, learns, and reasons about science. The goal is to develop a "knowledgeable machine" about science-where the system is able not just to retrieve answers from texts, but also to have a deeper understanding of the world and a capability to demonstrate understanding through question answering and explanation. ${ }^{13}$

Aside from developing deep learning systems, AI2 also has an incubator program to support and launch AI-fueled start-ups. In October 2017, it accepted Blue Canoe Learning as one of the first AI companies to join its incubator program. Using speech recognition and machine learning, the Seattle-based Canoe Learning has successfully developed an app to help users learn how to speak English. The collaboration's

\footnotetext{
${ }^{10} \mathrm{Kim}$ and Lee (n.d.). op. cit.

${ }^{11}$ Ibid.

${ }^{12}$ National Association of Securities Dealers Automated Quotations, a global electronic marketplace for buying and selling securities.

${ }^{13}$ Allen Institute for Artificial Intelligence. (n.d.). Aristo: Building Machines that read, learn, and reason. Retrieved from AI2 Website: https://allenai.org/aristo/ (https://allenai.org/aristo/).
} 
objective is to enhance this technology to help non-native English speakers improve their pronunciation. ${ }^{14}$

\section{California Community College System's “Doing What Matters"}

In California, the community college system is implementing a program named "Doing What Matters." It is a sophisticated enterprise resource planning system that takes and analyzes regional job market data to help colleges plan local programs and courses (DWM 2018). The initiative uses big data to timely link geolocationspecific job needs with precise job skill training initiatives through tailored degree or just-in-time micro-credential programs. In this scenario, AI services are also able to advise instructors in designing effective lesson plans (especially in math and science courses) for diverse groups of learners with different needs so that students can individually strengthen their skills at their own pace (Ascione 2017 as cited in Kim and Lee n.d.). ${ }^{15}$

\section{Stanford Mobile Inquiry-based Learning Environment (SMILE)}

The Stanford Mobile Inquiry-based Learning Environment (SMILE) is an assessment/inquiry maker that allows students to quickly create own inquiries or homework items based on their own learning for the day. ${ }^{16}$ As the application enables homework generation, completion, and competition games during class, it offers students the opportunity to review their lessons and create their own inquiries from them. This fosters a learning environment where students are not only engaged in discussing course topics, but are also enjoined to generate challenging questions that trigger higher order thinking. ${ }^{17}$

This education project has great potential for changing the landscape of generating and answering higher order thinking questions. Implemented in more than 30 countries, SMILE is collecting various types and levels of questions from learners in all types of education and training scenarios. As more higher order thinking questions are collected and discussed through the system, it becomes more possible to develop

\footnotetext{
${ }^{14}$ Soper (2017). New incubator program at Paul Allen's AI institute accepts English learning startup. Retrieved from https://www.geekwire.com/2017/new-incubator-program-paul-allens-ai-instit ute-accepts-english-learning-startup/.

${ }^{15} \mathrm{Kim}$ and Lee (n.d.). op. cit.

${ }^{16}$ Stanford School of Graduate Education. (n.d.) SMILE: Stanford Mobile Inquiry-based Learning Environment. Retrieved from Stanford GSE Website: https://gse-it.stanford.edu/smile.

${ }^{17}$ Kim and Lee (n.d.). op. cit.
} 
more accurate and intelligent services (digital assistants or AI-backed personalized tutors) that are able to deal with a much higher level and complex questions in Massive Open Online Course (MOOC) learning environments in the near future. ${ }^{18}$

\section{Application of These Good Practices or Examples}

The opportunity to leverage such models in learning systems, especially in online/distance education and MOOCs, is very promising. While AI research and its integration in education systems are not yet as successful, scalable, and accessible as we would like, continuous research and development of such initiatives may eventually significantly transform education systems on a massive scale.

As these practices are mostly pursued and successful in highly developed countries, international collaborations (among governments, international universities, and AI-supportive institutions) would be crucial for developing countries to be able to replicate them. Most of these models thrived because of partnerships and investments that fostered long-term research and development.

\section{Implication for the Future/Moving Forward}

While the difference that AI may present today may still seem miniscule or even undetectable, the potential that it may bring to the global education ecosystem will become much more transformative and widespread in an accelerating manner. Future learning environments will evolve quite rapidly to better understand and support learners at all stages of their learning cycles. At the same time, future learning models could be redesigned to move away from a knowledge dissemination system and toward a more knowledge co-creation system or environment (Peters and Besley 2017 as cited in as cited in Kim and Lee n.d.) that is intelligent enough to accommodate individual learning needs and goals as they are pursuing higher order learning opportunities on a massive scale. These innovations, when fully developed and made widely accessible, are expected to be embraced by educators.

Although such developments have positive impacts on enhancing education systems, they will also bring major challenges, particularly in ensuring that teachers are capable to quickly adapt to new teaching approaches and instructional technologies. This would entail extensive preparation, training, and, possibly, significant cultural shifts in classrooms (as cited in Kim and Lee n.d.). Ensuring data privacy and security is also expected to be a challenge, given inequalities of access to data and relevant technologies even among countries. Relatedly, given existing inequalities in access to Information and Communication Technology (ICT) infrastructure

\footnotetext{
${ }^{18}$ Ibid.
} 
and technologies among many countries and regions, the digital divide may worsen globally with the rapid advancement of AI and other technologies.

Finally, these challenges caused by anticipated advancements in technologies also spur the discussion on rethinking the kind of skills that education systems should be cultivating. For instance, there have been many discussions suggesting that one way of preparing learners for the future is by teaching them to code at an early age. Teaching children how to code has become a trend not only to steer STEM interest, but also because the huge potential of computational thinking in achieving holistic learning is being recognized-coding fosters logic, sequential thinking, curiosity, and problem-solving among learners. Such an approach, if integrated appropriately in the curriculum, could be instrumental in producing innovative learners with the ability to develop societal solutions for a better and more sustainable world.

\section{Conclusion}

Given the anticipated impacts of the Fourth Industrial Revolution, it is important to also direct discussion about how AI and other technologies' substantial strides in education systems to ensure that anticipated challenges are addressed, and how opportunities will be timely taken advantage of. Governments, especially those lagging behind, will have to pursue aggressive partnerships and increase investments in science, technology, research, and innovation to be able to keep up. Consequently, protecting data privacy and intellectual property rights also need to be ensured.

Education and training systems are also pressured to prepare learners for the future and to ensure that they have the skills to adapt - twenty-first century skills (a combination of foundational literacies, competencies, and character qualities), flexibility, and the capacity to innovate. While pursuing advancements in technologies such as $\mathrm{AI}$ and leveraging their potential to enhance education systems are also critical, the key to preparing for the future is fostering an attitude among people to continuously reboot thinking skills and pursue lifelong learning. Learners have to be encouraged and trained to ask questions, as this is where great innovations spring from.

Link to the presentation material: https://events.development.asia/materials/201 71212/entrepreneurship-education-industry-and-fourth-industrial-revolution.

\section{References}

Allen Institute for Artificial Intelligence. (n.d.). Aristo: Building Machines that read, learn and reason. Retrieved from AI2. https://allenai.org/aristo/.

Ascione, L. (2017). New AI tool helps teachers tackle math. Retrieved from Eschoolnews. https:// www.eschoolnews.com/2017/10/12/ibm-ai-tool-teachers-tackle-math/2/?all. 
DWM. (2018). Innovate what matters for jobs and the economy. Doing what matters. Retrieved from http://doingwhatmatters.cccco.edu/Overview/DWMFramework/Innovate.aspx.

Goel, A. K., \& Polepeddi, L. (2016). Jill Watson: A virtual teaching assistant for online education. Georgia Institute of Technology. https://smartech.gatech.edu/handle/1853/59104.

IBM. (nd). Teacher advisor with Watson. Retrieved from https://www.ibm.com/ibm/responsibility/ initiatives/activitykits/teacheradvisor/.

Jing, M. (2017). China wants to bring artificial intelligence to its classrooms to boost its education system. Retrieved from https://www.scmp.com/tech/science-research/article/2115271/ china-wants-bring-artificial-intelligence-its-classrooms-boost.

Kim, P. \& Lee, J. (n.d.). Evolution of online learning environments and the emergence of intelligent MOOCs.

Lipko, H. (2016). Meet Jill Watson: Georgia Tech's first AI teaching assistant. Retrieved from Georgia Tech. https://pe.gatech.edu/blog/meet-jill-watson-georgia-techs-first-ai-teachingassistant.

Peters, M., \& Besley, T. (2017). Co-creation in higher education students and educators preparing creatively and collaboratively to the challenge of the future. https://www.sensepublishers.com/ media/3279-co-creation-in-higher-education.pdf.

Soper, T. (2017). New incubator program at Paul Allen's AI institute accepts English learning startup. Retrieved from https://www.geekwire.com/2017/new-incubator-program-paul-allens-aiinstitute-accepts-english-learning-startup/.

Stanford School of Graduate Education. (n.d.) SMILE: Stanford Mobile Inquiry-based Learning Environment. Retrieved from Stanford GSE. https://gse-it.stanford.edu/smile.

The views expressed in this Chapter are those of the authors and do not necessarily reflect the views and policies of the Asian Development Bank (ADB) or its Board of Governors or the governments they represent.

ADB does not guarantee the accuracy of the data included in this Chapter and accepts no responsibility for any consequence of their use. The mention of specific companies or products of manufacturers does not imply that they are endorsed or recommended by ADB in preference to others of a similar nature that are not mentioned.

By making any designation of or reference to a particular territory or geographic area, or by using the term "country" in this Chapter, ADB does not intend to make any judgments as to the legal or other status of any territory or area.

This work is available under the Creative Commons Attribution 3.0 IGO license (CC BY 3.0 IGO) https://creativecommons.org/licenses/by/3.0/igo/. By using the content of this Chapter, you agree to be bound by the terms of this license. For attribution, translations, adaptations, and permissions, please read the provisions and terms of use at https://www.adb.org/terms-use\#openac cess.

This CC license does not apply to non-ADB copyright materials in this Chapter. If the material is attributed to another source, please contact the copyright owner or publisher of that source for permission to reproduce it. ADB cannot be held liable for any claims that arise as a result of your use of the material.

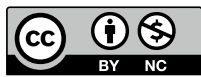

